\title{
Three prevalent mutations in a patient with phenylalanine hydroxylase deficiency: implications for diagnosis and genetic counselling
}

\author{
Per Guldberg, Harvey L Levy, Karen Friis Henriksen, Flemming Güttler
}

\begin{abstract}
Mutation analysis in a patient with mild hyperphenylalaninaemia showed three distinct base substitutions in exon 12 of the phenylalanine hydroxylase (PAH) gene. All three mutations, R413P, Y414C, and D415N, have previously been described as being independently associated with PAH deficiency. Family studies and independent analysis of the PAH alleles of the patient showed cosegregation of the R413P and Y414C mutations. Data on the ethnic background of the family provide evidence that the R413P mutation has occurred on a PAH allele carrying the Y414C mutation. Using current methods for mutation identification, the presence of two known mutations on a single PAH allele implies the risk of misdiagnosis of PAH deficiency and complicates genetic counselling. Our results stress the need for comprehensive mutation scanning of the PAH gene in diagnostic settings.

( $f$ Med Genet 1996;33:161-164)
\end{abstract}

Key words: phenylalanine hydroxylase deficiency; phenylketonuria; hyperphenylalaninaemia.

The liver enzyme phenylalanine hydroxylase (PAH, EC 1.14.16.1) catalyses the irreversible conversion of L-phenylalanine to L-tyrosine. Deficiency of PAH activity leads to hyperphenylalaninaemia and, without dietary treatment, usually causes mental retardation. The degree of enzyme impairment is highly variable. This is reflected in a broad span of biochemical and clinical phenotypes, ranging from classical phenylketonuria (PKU) to mild hyperphenylalaninaemia (MHP). ${ }^{1-4}$

Cloning and sequencing of the PAH gene ${ }^{5}$ has enabled potential disease causing mutations in hyperphenylalaninaemic patients to be identified. More than 200 different mutations have been reported, the majority of which are single base substitutions and microdeletions. ${ }^{6}$ Despite this extensive genetic variation, many $\mathrm{PAH}$ deficient patients share the same combination of mutations owing to enrichment of certain mutations through founder events. Prevalent mutations include R408W, IVS12nt1g $\rightarrow$ a, IVS10nt $-11 \mathrm{~g} \rightarrow \mathrm{a}$, and $\mathrm{Y} 414 \mathrm{C}$ in European populations, and R413P and IVS4nt $-1 \mathrm{~g} \rightarrow \mathrm{a}$ in Oriental populations. ${ }^{7}$ This particular distribution of mutations allows for the con- centration of patients with identical genotypes in certain geographical areas, thus providing the basis for establishing genotype-phenotype correlations by comparing clinical parameters among patients with identical PAH mutation genotypes. $^{89}$

Improved methods of detection and identification of mutations have made DNA analysis a powerful tool for diagnosis of $\mathrm{PAH}$ deficiency. ${ }^{10}$ In routine diagnostic settings, the identification of two known mutations in a newborn with raised blood levels of phenylalanine would generally be sufficient to establish PAH deficiency as the cause of hyperphenylalaninaemia, and to predict the metabolic phenotypic outcome. ${ }^{4}$ This is not always true, however, and in this paper we describe a patient with hyperphenylalaninaemia who has three known mutations. The implications of this observation for molecular diagnosis and genetic counselling are discussed.

\section{Materials and methods}

SAMPLES

The proband is a 13 year old girl with MHP. She was born after an uneventful pregnancy by normal, term, spontaneous delivery. Her birth weight was $2980 \mathrm{~g}$. She was breast fed and had a normal neonatal course. Her newborn blood screen at the age of 3 days indicated a phenylalanine level of $600 \mu \mathrm{mol} / \mathrm{l}(10 \mathrm{mg} / \mathrm{dl})(\mathrm{de}-$ termined by the Guthrie bacterial inhibition assay $\left.^{11}\right)$. Confirmatory plasma amino acid analysis at 7 days disclosed a phenylalanine level of $290 \mu \mathrm{mol} / 1(4.8 \mathrm{mg} / \mathrm{dl})$. Dietary treatment was not recommended. Follow up evaluations have shown normal growth and development. Plasma levels on a normal diet have ranged from $220 \mu \mathrm{mol} / 1 \quad(3.7 \mathrm{mg} / \mathrm{dl})$ to $410 \mu \mathrm{mol} / 1$ $(6.8 \mathrm{mg} / \mathrm{dl})$. At the age of 6 years her IQ was 109 (determined by the McCarthy Scales of Children's Abilities). She is an excellent student. Her immediate family consists of normal parents and four normal sibs, all of whom have normal blood phenylalanine levels of $<80 \mu \mathrm{mol} / 1$.

\section{MUTATION ANALYSIS}

Genomic DNA was isolated from EDTA anticoagulated blood. Scanning for mutations in the PAH gene was performed by PCR in combination with "broad range" DGGE as de- 


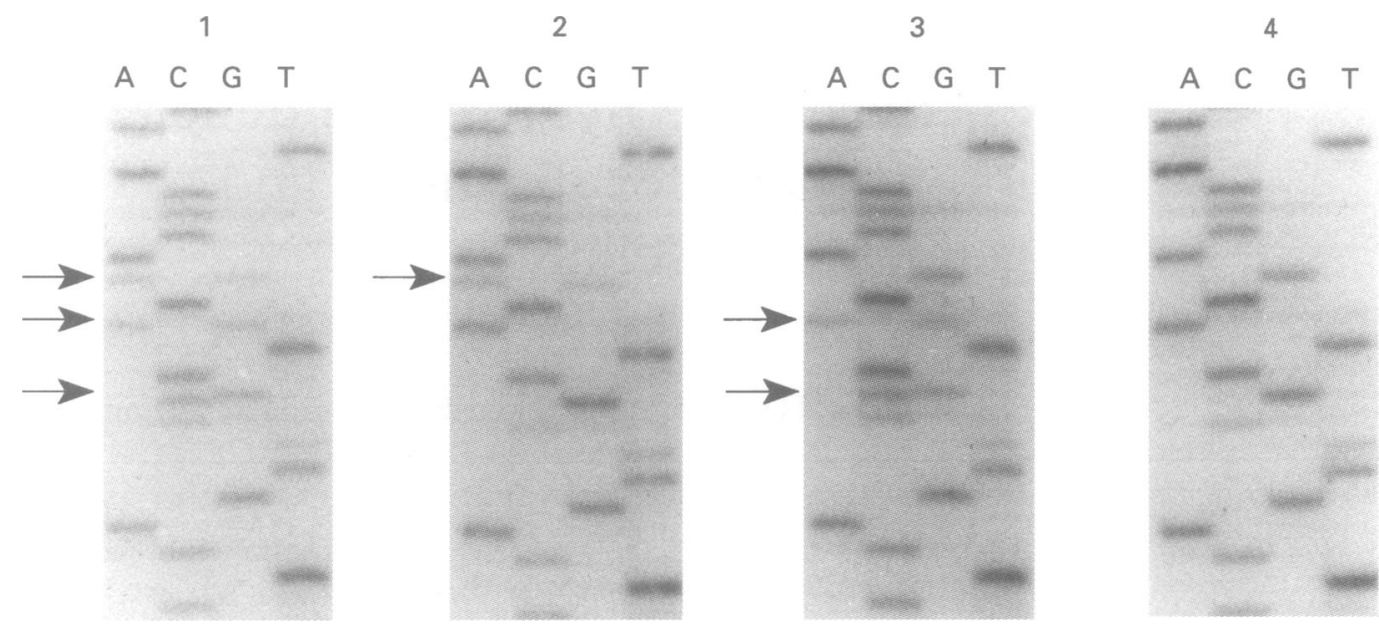

Figure 1 Partial sequence ladders showing exon 12 of the PAH gene. Arrows indicate sites of mutation. Lane 1 , proband carrying the $R 413 P(G \rightarrow C), Y 414 C(A \rightarrow G)$, and $D 415 N(G \rightarrow A)$ mutations. Lane 2 , mother carrying the D415N mutation. Lane 3, father carrying the R413P and Y414C mutations. Lane 4, normal control.

scribed elsewhere. ${ }^{12}$ Sequences of GC clamped primers for amplification of all $13 \mathrm{PAH}$ coding exons and their flanking intronic junctions have previously been reported. ${ }^{13}$ Exonic regions with positive DGGE analyses were subjected to direct sequencing as described. ${ }^{14} 15$ For mutation analysis of individual alleles of exon 12 of the PAH gene, homoduplex bands were excised from the denaturing gradient gel and incubated over night in $100 \mu \mathrm{l}$ of water at $4^{\circ} \mathrm{C}$. After

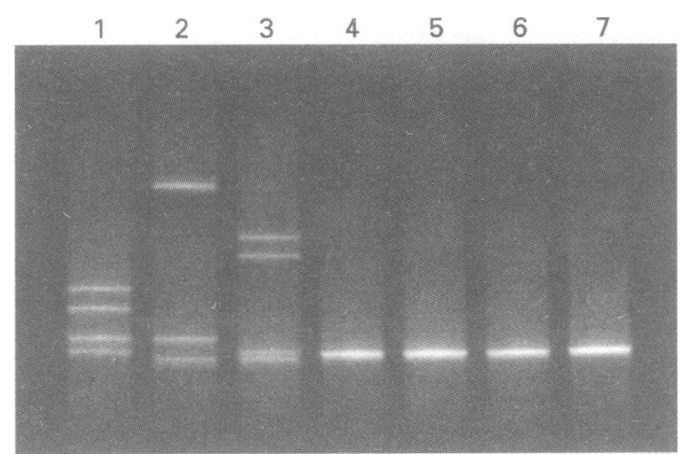

Figure 2 Family analysis showing mutations in exon 12 of the PAH gene. Exon 12 was PCR amplified and subjected to DGGE analysis. The presence of one or more mutations is indicated by the presence of more than one band. Lane 1, mother (genotype D415N/N). Lane 2, proband (genotype R413P-Y414C/D415N). Lane 3, father (genotype R413P-Y414C/N). Lanes 4-7, sibs (genotype N/N).

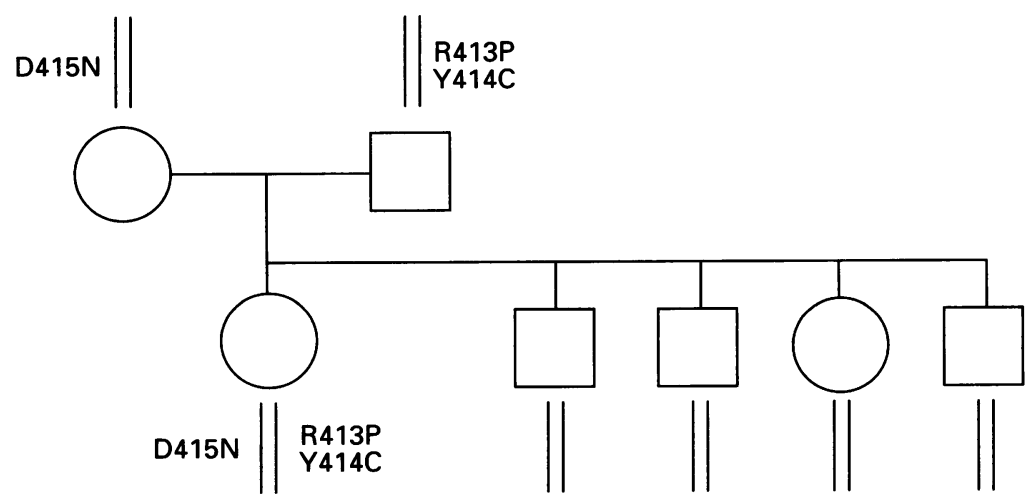

Figure 3 Family pedigree showing the segregation of three mutations (R413P, Y414C, and $\mathrm{D} 415 \mathrm{~N}$ ) on two alleles. a brief centrifugation, the eluate was diluted 10000 -fold, and $1 \mu \mathrm{l}$ of this dilution was used as template in PCR. Sequencing of the double stranded PCR product was performed as indicated above.

\section{Results}

Comprehensive mutation scanning of the PAH gene of the proband by means of PCR in combination with "broad range" DGGE" showed a composite pattern of homoduplex and heteroduplex bands in exons $4,6,7$, and 12 (data not shown), indicating the presence of heterozygous mutations. Direct sequence analysis of exons 4,6 , and 7 showed heterozygosity for the common silent mutations IVS3nt-22t/c, Q232Q, and V245V. No other sequence alterations were found in these regions. In exon 12, three distinct missense mutations involving three successive codons were identified: a $G$ to $C$ transversion at codon 413 , causing the substitution of proline for arginine; an $A$ to $G$ transition at codon 414 , causing the substitution of cysteine for tyrosine; and a G to A transition at codon 415 , causing the substitution of asparagine for aspartic acid (fig 1).

To determine which of the three mutations were transmitted in cis, individual alleles of exon 12 were recovered from the denaturing gradient gel and subjected to sequence analysis. By this approach, the presence of R413P and Y414C in cis could be established. Sequence analysis of DNA from the parents showed that the R413P-Y414C double substitution was inherited on the paternal mutant chromosome, whereas the $\mathrm{D} 415 \mathrm{~N}$ mutation was segregating on the maternal mutant chromosome (fig 1). Neither of the mutations was found in the four healthy sibs (fig 2). A summary of the pedigree analysis is shown in fig 3 .

\section{Discussion}

We describe a child with PAH deficient MHP in which three distinct missense PAH mutations segregate on two mutant PAH alleles. All three mutations have previously been shown to se- 
gregate independently and their individual associations with PAH deficiency are well established. The R413P mutation accounts for $14 \%$ of northern Chinese and $27 \%$ of Japanese PKU alleles. The mutation results in negligible enzymatic activity when expressed in heterologous mammalian cells, which is compatible with the classical PKU phenotype observed in R413P homozygotes. ${ }^{16}$ The Y414C mutation is a prevalent mutation in Europe. It is associated with a mild PKU phenotype and results in $50 \%$ residual enzyme activity in vitro. ${ }^{817}$ The $\mathrm{D} 415 \mathrm{~N}$ mutation has been identified in several European populations as a common cause of $\mathrm{MHP},{ }^{18}$ although its impact on enzyme activity has not been evaluated in vitro. In the hyperphenylalaninaemic patient described in this paper, the R413P and Y414C mutations were inherited in cis, and the $\mathrm{D} 415 \mathrm{~N}$ mutation was inherited in trans. This pattern of inheritance is compatible with the MHP phenotype in the proband owing to the dominance of the "mild" allele (D415N) over the potentially "severe" allele (R413P-Y414C).

The origin of the R413P-Y414C allele is not clear. The location of the two base substitutions, separated by only two base pairs, renders recombination highly unlikely. Since both mutations occur on haplotype $4,{ }^{1617}$ it is not possible to determine the nature of the origin of the R413P-Y414C allele by molecular means. The proband's father, who carries the R413P-Y414C allele, however, is of European descent, indicating that the original chromosome probably carried the Y414C mutation and the double mutation resulted from occurrence of the R413P mutation. The hypermutability hypothesis supports this suggestion. In contrast to the Y414C mutation, the R413P mutation involves a $\mathrm{CpG}$ dinucleotide, which has a 8.5 times higher mutation rate than that of the average dinucleotide ${ }^{19}$ and is known to be a mutation "hot spot" in a wide range of human genes, including the $\mathrm{PAH}$ gene. ${ }^{20}$

The frequency of the R413P-Y414C allele in European populations is not known. Hitherto, the majority of Y414C alleles have been identified by searching for this mutation using diagnostic mutation detection techniques, for example, allele specific oligonucletide (ASO) probing, or digestion with restriction endonucleases. ${ }^{21}$ These methods do not provide information about other sequence alterations in the PAH gene and will generally not allow recognition of the $\mathrm{R} 413 \mathrm{P}$ mutation. Re-examination of $\mathrm{Y} 414 \mathrm{C}$ bearing chromosomes by sequence analysis would determine the actual frequency of the R413P-Y414C allele. Although this could turn out to be a frequent PKU allele in some populations, investigation of more than 60 European chromosomes carrying the $\mathrm{Y} 414 \mathrm{C}$ mutation has not revealed cosegregation with the R413P mutation in any case (unpublished results), indicating that the R413P-Y414C allele is probably rare in Europe. This is in agreement with previous results, showing the absence of the R413P mutation from 50 European mutant chromosomes. ${ }^{16}$ The only demonstration of the R413P mutation in Europe is from The Netherlands, ${ }^{6}$ but no indication of its frequency or its possible cosegregation with the Y414C mutation has been reported.

As indicated above, the R413P mutation is associated with classical PKU, while the Y414C mutation is associated with mild PKU. Therefore, coinheritance of the two mutations in trans would be expected to result in a mild PKU phenotype, and a phenylalanine restricted diet would be implemented. In contrast, the phenotypic outcome in a patient with the R413P and Y414C mutations inherited in cis is strongly dependent on the mutation inherited in trans. If the latter mutation is associated with classical PKU, a severe phenotype results and requires rigorous treatment, whereas if it is associated with $\mathrm{MHP}$, as in the present case, the phenotypic outcome is MHP with no need for treatment. Consequently, accurate prognosis and considerations of therapy require complete genotyping and correct interpretation of the genotype. Current methods for detection and identification of point mutations are based on PCR amplification of target DNA and will not always allow discrimination between the two alleles. ${ }^{2122}$ With no consideration of the possibility that two causative mutations exist on a single allele, the search for mutations will generally be stopped once two known causative mutations have been identified. The presence of a double mutant allele may lead to misinterpretation of genotype data, which can have adverse consequences to patients with PAH deficiency and their families.

The prevalence of more than one mutation on a single allele of a disease gene has not yet been determined, but may constitute a problem in molecular diagnosis. Double mutant alleles have previously been observed in various mendelian disorders, such as Gaucher disease, ${ }^{23}$ cystic fibrosis, ${ }^{24}{ }^{25}$ PKU, ${ }^{915}$ and galactosaemia. ${ }^{26}$ To minimise the risk of misdiagnosis of $\mathrm{PAH}$ deficiency and other autosomal recessive disorders, the entire disease associated gene should be scanned for mutations, in particular where family analysis cannot be performed.

We wish to thank the proband and her family for their coWerative to thank the proband and her family for their cooperative participation in this study. PG is supported by Felstudy is supported by The Danish Medical Research Council study is supported by The Danish Medical Research Council BIOMED I (Area 3: Human Genome Analysis), The Danish BIOMED I (Area 3: Human Genome Analysis), The Danish Biotechnological Research and Development Programme 19911995 (Grant 5.18.03), The Danish Health Insurance FoundNovo Foundation, The Foundation of 1870 , Franz Hoffmann's Memorial Fund, and Emil C Hertz's Fund.

1 Kaufman S. Phenylketonuria: biochemical mechanisms. In Agranoff BW, Aprison MH, eds. Advances in neuro chemistry. Vol 2. New York: Plenum Press, 1976:1-132.

Scriver CR, Kaufman S, Eisensmith RC, Woo SLC. The hyperphenylalaninemias. In: Scriver CR, Beaudet AL, Sly $\mathrm{WS}$, Valle $\mathrm{D}$, eds. The metabolic and molecular bases of inherited disease. New York: McGraw-Hill, 1995:1015-75.

3 Güttler F. Hyperphenylalaninemia: diagnosis and classification of the various types of phenylalanine hydroxylase deficiency in childhood. Acta Paediatr Scand 1980;supp 280:1-80.

4 Güttler F, Guldberg P. Mutations in the phenylalanine hydroxylase gene: genetic determinants for the phenotypical heterogeneity of hyperphenylalaninemia. Acta Paediatr Scand 1994;suppl 407:49-56.

5 Kwok SCM, Ledley FD, DiLella AG, Robson KJH, Woo SLC. Nucleotide sequence of a full length complementary DNA clone and amino acid sequence of human phenylalanine hydroxylase. Biochemistry 1985;24:556-61. 
6 PAH Mutation Analysis Consortium. July 1995 Edition. Montreal: McGill University, 1995.

7 Eisensmith RC, Woo SLC. Population genetics of phenylketonuria. Acta Paediatr Scand 1994;suppl 407:19-26.

8 Okano Y, Eisensmith RC, Güttler F, et al. Molecular basis of phenotypic heterogeneity in phenylketonuria. $N$ Engl $\mathcal{F}$ Med 1991;324:1232-8.

9 Svensson E, von Döbeln U, Eisensmith RC, Hagenfeldt L, Woo SLC. Relation between genotype and phenotype in Swedish phenylketonuria and hyperphenylalaninemia patients. Eur $\mathcal{F}$ Pediatr 1993;152:132-9.

10 Guldberg P, Güttler F. Mutations in the phenylalanine hydroxylase gene: methods for their characterization. Acta Paediatr Scand 1994;suppl 407:27-33.

11 Guthrie R, Susi A. A simple phenylalanine method for detecting phenylketonuria in large populations of newborn infants. Pediatrics 1963;32:338-43

12 Guldberg P, Güttler F. 'Broad-range' DGGE for single-step mutation scanning of entire genes: application to human phenylalanine hydroxylase gene. Nucleic Acids Res 1994; 22:880-1.

13 Guldberg P, Romano V, Ceratto N, et al. Mutational spectrum of phenylalanine hydroxylase deficiency in Sicily: trum of phenylalanine hydroxylase deficiency in Sicily: implications for diagnosis of hyperphenylalaninem

14 Wong C, Dowling CE, Saiki RK, et al. Characterization of $\beta$-thalassaemia mutations using direct genomic sequencing of amplified single copy DNA. Nature 1987;330:384-6.

15 Guldberg P, Henriksen KF, Güttler F. Molecular analysis of phenylketonuria in Denmark: $99 \%$ of the mutations detected by denaturing gradient gel electrophoresis. Genomics 1993;17:141-6.
16 Wang T, Okano Y, Eisensmith RC, et al. Founder effect of a prevalent phenylketonuria mutation in the Oriental population. Proc Natl Acad Sci USA 1991;88:2146-50.

17 Okano Y, Eisensmith RC, Dasovich M, et al. A prevalent missense mutation in Northern Europe associated with hyperphenylalaninemia. Eur $\mathcal{F}$ Pediatr 1991;150:347-52.

18 Economou-Petersen E, Henriksen KF, Guldberg P, Güttler phenylalaninemia. Genomics 1992;14:1-5.

19 Cooper DN, Krawczak M. Human gene mutation. Oxford: BIOS Press, 1993.

20 Abadie V, Lyonnet S, Maurin N, et al. CpG dinucleotides are mutation hot spots in phenylketonuria. Genomics 1989; 5:936-9.

21 Cotton RGH. Current methods of mutation detection. $M u$ tat Res 1993;285:125-44.

22 Grompe $M$. The rapid detection of unknown mutations in nucleic acids. Nature Genet 1993;5:111-17.

23 Latham T, Grabowski GA, Theophilus BDM, Smith FI. Complex alleles of the $\beta$-glucosidase gene in Gaucher disease. Am 7 Hum Genet 1990;47:79-86.

24 Dörk T, Wulbrand U, Richter T, et al. Cystic fibrosis with three mutations in the cystic fibrosis transmembrane conductance regulator gene. Hum Genet 1991;87:441-6.

25 Savov A, Angelicheva D, Balassopoulou A, et al. Double mutant alleles: are they rare? Hum Mol Genet 1995;4: $1169-71$.

26 Elsas LJ, Langley S, Steele E, et al. Galactosemia: a strategy to identify new biochemical phenotypes and molecular genotypes. Am $\mathcal{F}$ Hum Genet 1995;56:630-9. 